\title{
Computation of a family of non-cosymmetrical equilibria in a system of nonlinear parabolic equations
}

\author{
K. Frischmuth* $\quad$ V. G. Tsybulin ${ }^{\dagger}$
}

June 14, 2002

\begin{abstract}
We study a system of two nonlinear parabolic equations with cosymmetry and find a continuous family of equilibria with nonconstant spectrum. Basing on the finite-difference approach, we develop a numerical method for solving the partial differential equations and calculating the continuous family of non-cosymmetrical equilibria. An application of the proposed system to population kinetics is demonstrated. Numerically computed families are presented. The dependence on model parameters is discussed.
\end{abstract}

Keywords: Cosymmetry, parabolic systems, stationary states

AMS Classification: 35B20, 65M06, 35K60, 73Q05, $90 A 16$

\section{Introduction}

Recently V. Yudovich $[1,2]$ introduced the notion of a cosymmetry in order to explain the onset of a continuous family of equilibria with variable spectra in certain problems of mathematical physics. At present, the theory of cosymmetry and its applications are intensively studied, cf. [1-10].

For a differential equation $\dot{u}=F(u)$ in a Hilbert space $H$, a cosymmetry is an non-trivial operator $L$ which is orthogonal to $F$ at each point of the phase space, i.e. $(F(u), L(u))=0$, where $(\cdot, \cdot)$ denotes the inner product. Assume a state of equilibrium $u_{0} \in H, F\left(u_{0}\right)=0$ to be non-cosymmetric, i.e. $L\left(u_{0}\right) \neq 0$. Then, as a consequence of the Implicit Function Theorem, cf. [1, 4], $u_{0}$ belongs to a continuous one-parameter family $\{u(\tau): F(u(\tau))=0, \tau \in \Gamma\}$ with $u(0)=u_{0}$, provided there are no additional degeneracies. In degenerate cases there exists a $k$-dimensional sub-manifold of equilibria that contains the equilibrium $u_{0}$.

We construct below such a system of nonlinear parabolic equations which admits a non-trivial cosymmetry and a family of non-cosymmetrical equilibria. Problems of that type were first observed and studied in mathematical physics, in particular in diffusionconvection problems in porous media. Numerical solutions for such systems with arbitrary

* Department of Mathematics, University of Rostock, Rostock - Germany, kurt.frischmuth@mathematik.uni-rostock.de

${ }^{\dagger}$ Department of Mathematics and Mechanics, Rostov State University, 344090 Rostov on Don - Russia, tsybulin@math.rsu.ru 
schemes usually do not find a continuous family of equilibria but rather a number of isolated equilibria.

We intend to model a situation in population kinetics where infinitely many stationary states are possible. We assume two populations sharing a common space, e.g. a river bank or a traffic route, and suppose a dominant role of the unique spatial coordinate $x$. Looking for best opportunities for their respective trades, each population may move in dependence on the current concentrations of their own kind and the other one. However, the first population (concentration function $u(x, t)$ ) thrives on relocation of the second $(v(x, t))$, while the second suffers from motion of the first.

Thus, we consider a system for a time dependent vector field $w(x, t)=(u(x, t), v(x, t))^{\top}$ defined on a domain $\Omega=[0, a] \subset \mathbb{R}$ with Dirichlet conditions applied on $\partial \Omega$

$$
\begin{aligned}
\dot{w} & =\epsilon w^{\prime \prime}+\kappa w^{\prime}+f\left(w, w^{\prime}\right), \\
w(x, 0) & =w_{0}(x) \text { on } \Omega, \\
w(x, t) & =0 \text { on } \partial \Omega \times[0, T] .
\end{aligned}
$$

Here the dot and prime mean differentiation with respect to time $t$ and space coordinate $x$. The solution is sought on the time span $T$, in particular $T$ may be $+\infty$. Square matrixes $\kappa$ and $\epsilon$ comprise the known constants $\epsilon_{u}, \epsilon_{v}, \kappa_{u}, \kappa_{v}$,

$$
\epsilon=\left(\begin{array}{cc}
\epsilon_{u} & 0 \\
0 & \epsilon_{v}
\end{array}\right), \quad \kappa=\left(\begin{array}{cc}
0 & \kappa_{u} \\
\kappa_{v} & 0
\end{array}\right)
$$

It is essential that the flux of the $v$-component results in a source of the $u$-component, and vice versa. Both sources may be interpreted as some preferred direction of a structured medium. It is of great interest to consider the case of opposite signs, i.e., when the product $\kappa_{u} \kappa_{v}$ is negative. Diffusion terms, given by second order derivatives (one-dimensional Laplace operator), often play merely the role of a regularization. In particular, the parameters $\epsilon_{u}, \epsilon_{v}$ may be important beyond their physical meaning. The nonlinear term $f=\left(f_{u}\left(w, w^{\prime}\right), f_{v}\left(w, w^{\prime}\right)\right)^{\top}$ describes some sort of activity depending simultaneously on transportation and availability of the components.

The system (1)-(3) with zero initial conditions has the trivial solution $w=0$. More interesting is that there also exist nontrivial stationary solutions for suitable values of the parameters $\kappa_{u}$ and $\kappa_{v}$ and certain types of nonlinearities. Here, we concentrate on the case when in fact a whole one-parameter family of stationary solutions exists, but they cannot be obtained by action of an invariance as translation or rotation. This is exactly the situation for which the theory of cosymmetry and the notion of families of non-cosymmerical equilibria were introduced, [1, 2].

In the next section, we are going to examine the above system and derive the conditions for a nontrivial cosymmetry. We identify parameters which give, indeed, a continuous family of equilibria, the calculation of which is presented in Sec. 4. To this end, we introduce a special numerical scheme which is shown to work well for the system (1)-(3).

\section{Preliminary Analysis of the System}

First, we briefly describe some essential properties of the underlying system. We define a Hilbert space $H=L^{2}(\Omega) \times L^{2}(\Omega)$ with the usual scalar product. 
In order to enhance the dissipativity of the system we assume that the functions $f_{u}, f_{v}$ are such that the following integral equality holds

$$
\int_{\Omega}\left(\kappa_{v} f_{u} u+\kappa_{u} f_{v} v\right) d x=0
$$

Then, multiplying the first equation (1) by $\kappa_{v} u$ and the second one by $\kappa_{u} v$, summing and integrating over the domain $\Omega$ we obtain

$$
\frac{d}{d t} \int_{\Omega}\left(\kappa_{v} u^{2}+\kappa_{u} v^{2}\right) d x=-\int_{\Omega}\left(\kappa_{v} \epsilon_{u} u^{\prime 2}+\kappa_{u} \epsilon_{v} v^{\prime 2}\right) d x
$$

One can see that in the case $\kappa_{u}=0$ and $\epsilon_{u}>0$ the dissipativity of the component $u(x, t)$ is guaranteed, and analogically, for $\kappa_{v}=0$ and $\epsilon_{v}>0$ we deduce the decrease of $\int_{\Omega} v^{2} d x$.

\subsection{Cosymmetry Condition}

We are interested in conditions for which the system (1)-(3) has a cosymmetry. We restrict ourselves to the case of a linear cosymmetry in the following form

$$
L(w)=\left(\begin{array}{cc}
0 & \epsilon_{v} \\
\epsilon_{u} & 0
\end{array}\right) w .
$$

In order to cover a reasonable class of nonlinearities, we assume $f_{u}$ and $f_{v}$ to be arbitrary bilinear forms in $w$ and $w^{\prime}$

$$
f_{u}=\epsilon_{u} w^{\top} M^{u} w^{\prime}, \quad f_{v}=\epsilon_{v} w^{\top} M^{v} w^{\prime} .
$$

Here the factors $\epsilon_{u}$ and $\epsilon_{v}$ are used for convenience of subsequent presentation. The following Lemma shows the existence of matrices that lead to a cosymmetry of the underlying problem.

Lemma: 1 Let the matrices in (8) be of the form

$$
M^{u}=\left(\begin{array}{cc}
\delta-2 \gamma & \beta \\
\alpha & \gamma-2 \delta
\end{array}\right), \quad M^{v}=\left(\begin{array}{cc}
\alpha-2 \beta & \gamma \\
\delta & \beta-2 \alpha
\end{array}\right)
$$

where the constants $\alpha, \beta, \gamma, \delta$ are free parameters. Then the system (1)-(3) has a cosymmetry (7) and the condition (5) is fulfilled.

The prove is done by direct verification. Let us multiply the equation (1) by $L(w)$, sum and integrate over the domain $\Omega$. One can easily check that the right-hand side of this equation vanishes, because the following equalities are valid

$$
\begin{aligned}
\int_{\Omega} \epsilon_{u} \epsilon_{v}\left(u^{\prime \prime} v-v^{\prime \prime} u\right) d x & =0 \\
\int_{\Omega}\left(\epsilon_{v} \kappa_{v} v^{\prime} v-\epsilon_{u} \kappa_{v} u^{\prime} u\right) d x & =0 \\
\int_{\Omega}\left(\epsilon_{v} f_{u} v-\epsilon_{u} f_{v} u\right) d x & =0 .
\end{aligned}
$$


This in turn is obtained using integration by parts, Green's formula and the boundary conditions. As result we have

$$
\int_{\Omega}\left(\epsilon_{v} \dot{u} v-\epsilon_{u} \dot{v} u\right) d x=0
$$

which by definition means that (7) is a cosymmetry of the system (1)-(3).

The validity of (5) follows from similar transformations.

So, the system (1)-(3) is a cosymmetric one for any values $\kappa_{u}, \kappa_{v}$ and the expressions for the nonlinearities based on the four-parametric family of matrices (9). We present below examples of parameter values such that the assumptions of Lemma 1 are satisfied, and compute a number of continuous families consisting of steady states.

Another case of nonlinearities which allows a cosymmetry is given by

$$
f_{u}=\epsilon_{u} \sum_{k=1}^{K} \mu_{k} u^{\alpha_{k}+1} v^{\beta_{k}}, \quad f_{v}=\epsilon_{v} \sum_{k=1}^{K} \mu_{k} u^{\alpha_{k}} v^{\beta_{k}+1},
$$

where for simplicity we will suppose $\alpha_{k} \geq 0, \beta_{k} \geq 0$, and $\mu_{k} \in \mathbb{R}$.

As a particular case the nonlinearities (14) may model logistic growth, if we specify

$$
f_{u}=\epsilon_{u} \mu u\left(1-\frac{u}{u_{\infty}}\right), \quad f_{v}=\epsilon_{v} \mu v\left(1-\frac{u}{u_{\infty}}\right) .
$$

Here $u_{\infty}$ plays the role of a limiting value on the concentration $u(x, t)$. It should be mentioned that the logistic law is applied only to the growth of the first population, whereas the other is regulated by closeness to $u_{\infty}$.

\subsection{Stability of zero equilibrium}

It is easy to analyze the stability of the cosymmetrical equilibrium $w=0$. Without loss of generality we take $\kappa_{u}>0$ and $\kappa_{v}<0$. Then, we change variables

$$
u=\tilde{u} \sqrt{\kappa_{u} \epsilon_{v}}, \quad v=\tilde{v} \sqrt{-\kappa_{v} \epsilon_{u}},
$$

and set

$$
\lambda=\frac{1}{2} \sqrt{\frac{-\kappa_{u} \kappa_{v}}{\epsilon_{u} \epsilon_{v}}} .
$$

For simplicity we take $\kappa_{u}=-\kappa_{v}=\lambda$. After that the corresponding spectral problem is given

$$
0=\tilde{u}^{\prime \prime}+2 \lambda \tilde{v}^{\prime}, \quad 0=\tilde{v}^{\prime \prime}-2 \lambda \tilde{u}^{\prime}, \quad \tilde{v},\left.\tilde{v}\right|_{\delta \Omega}=0 .
$$

We introduce the complex function $W=\tilde{u}+i \tilde{v}$ and rewrite (18) as

$$
0=W^{\prime \prime}-2 \lambda i W^{\prime},\left.\quad W\right|_{\delta \Omega}=0,
$$

Finally, we substitute $W=U \exp (i \lambda x)$ and deduce

$$
0=U^{\prime \prime}+\lambda^{2} U,\left.\quad U\right|_{\delta \Omega}=0,
$$

So, we find that $\lambda_{j}=j \pi / a, j \in \mathbb{Z}$ and all eigenvalues are simple [11].

Because the original problem is equivalent to the eigenvalue problem for the $1 \mathrm{~d}$ Laplace 
operator corresponding to pairs of functions, the multiplicity of each eigenvalue doubles. Thus the critical value is $\lambda_{\text {crit }}=\pi / a$, and at $\lambda>\lambda_{\text {crit }}$ the zero equilibrium becomes unstable.

In the case of nonlinearities given by (15) similar manipulations give the following result

$$
\lambda_{j}=\sqrt{\frac{j^{2} \pi^{2}}{a}-\mu} .
$$

\section{Solution method}

We apply the method of lines to the solution of the system (1)-(3). To discretize the right-hand side we use second order finite difference operators, paying special attention to the approximation of nonlinear terms. It is a crucial point for systems with cosymmetry: an inappropriate approximation may lead to the loss of the cosymmetry [6]. As result, one may obtain a number of isolated equilibria instead of a continuous family of steady states.

\subsection{Semi-discretization}

We consider the case of a uniform grid on $\Omega=[0, a]$

$$
x_{j}=j h, \quad j=0, \ldots, N+1, \quad h=a /(N+1) .
$$

All unknowns are defined at the nodes $x_{j}$, and the notations $u_{j}$ and $v_{j}$ are used for discrete values. The discretized boundary conditions take the following form

$$
u_{0}=v_{0}=u_{N+1}=v_{N+1}=0 .
$$

For first order derivatives and the Laplacian we use centered difference operators

$$
D_{j}^{1}(u)==\frac{u_{j+1}-u_{j-1}}{2 h}, \quad D_{j}^{2}(u)=\frac{u_{j+1}-2 u_{j}+u_{j-1}}{h^{2}} .
$$

Then, the discrete analog of the system (1) is given as

$$
\begin{aligned}
& \dot{u}_{j}=\epsilon_{u} D_{j}^{2}(u)+\kappa_{u} D_{j}^{1}(v)+f_{u, j}, \quad j=0, \ldots, N, \\
& \dot{v}_{j}=\epsilon_{v} D_{j}^{2}(v)+\kappa_{v} D_{j}^{1}(u)+f_{v, j} .
\end{aligned}
$$

The nonlinear terms $f_{u, j}$ and $f_{v, j}$ are defined below. Using the discrete version of Green's formulae and reordering the sum (discrete analog of integration by parts) we conclude that the following equalities are valid

$$
\begin{array}{r}
h \epsilon_{v} \epsilon_{u} \sum_{j=1}^{N}\left(D_{j}^{2}(u) v_{j}-D_{j}^{2}(v) u_{j}\right)=0 \\
\sum_{j=1}^{N}\left(\epsilon_{v} \kappa_{v} D_{j}^{1}(v) v_{j}-\epsilon_{u} \kappa_{v} D_{j}^{1}(u) u_{j}\right)=0 .
\end{array}
$$


The given relations are the analogs of equalities (10) and (11), respectively. Thus, the discrete analog of (7) is a cosymmetry for (24)-(25) iff the following equality is fulfilled

$$
h \sum_{j=1}^{N}\left(\epsilon_{v} f_{u, j} v_{j}-\epsilon_{u} f_{v, j} u_{j}\right)=0 .
$$

The approximation of the nonlinear terms (14) can be done in a straightforward way. A special approximation has been developed to deal with the nonlinear terms (8) which depend both on the function values and their slopes. We apply for this purpose the direct method of free parameters, doing the calculations by the computer algebra system Maple.

The general second order accurate finite-difference operator on a three nodes stencil may be written as

$$
\left.u^{\prime} v\right|_{x_{j}} \approx D_{j}(u, v) \equiv \sum_{k, l=-1}^{1} p_{k l} u_{j+k} v_{j+l} .
$$

Here $p_{k l}, k, l=1,2,3$ are unknown or free parameters.

We compute a Taylor series for the expression $\left.u^{\prime} v\right|_{x_{j}}-D_{j}(u, v)$ and demand terms up to third order on $h$ to vanish. Thus, we derive a system of six linear equations for the $p_{k l}$ and then solve it. As result we obtain a three-parameter family of operators giving second order accuracy for the term $u^{\prime} v$.

To establish an additional relation on the unknown parameters we impose the integral equality

$$
\int_{0}^{a} u^{\prime} u \phi(u) d x=0
$$

This is valid, in particular, for homogeneous boundary conditions.

The discrete analog of (30) must be satisfied at least for some functions $\phi(u)$

$$
h \sum_{j=1}^{N} D_{j}(u, u) \phi\left(u_{j}\right)=0 .
$$

We get two additional equations on the parameters $p_{k l}$ from this requirement for $\phi(u)=u$.

Eventually, we find a one-parameter formula approximating $u^{\prime} v$

$$
\begin{aligned}
D_{j}(u, v)= & \frac{1}{6 h}\left[(1+\zeta)\left(u_{j+1}-u_{j-1}\right) v_{j}-\zeta u_{j}\left(v_{j+1}-v_{j-1}\right)\right. \\
& \left.+u_{j+1} v_{j+1}-u_{j-1} v_{j-1}+(1-\zeta)\left(u_{j+1} v_{j-1}-u_{j-1} v_{j+1}\right)\right] .
\end{aligned}
$$

Finally, from the cosymmetry relation (28) we deduce that only for $\zeta=1$ all requirements are met.

We remark that more examples of the application of computer algebra systems to the derivation of numerical schemes are given, for instance, in [12].

Let us rewrite the system of ordinary differential equations (24)-(25) as follows

$$
\dot{Y}=(A+\lambda B) Y+F(Y) \text {. }
$$

Here $A$ is a positive-definite matrix, $B$ is a skew-symmetric matrix, and $F(Y)$ denotes a nonlinear term. The vector $\mathrm{Y}$ is given by

$$
Y=\left(u_{1}, \ldots, u_{N}, v_{1}, \ldots, v_{N}\right) .
$$


Note that (33) is a vector equation in $\mathbb{R}^{2 N}$, where $N$ is the number of inner grid points in $\Omega$.

Without loss of generality we consider further $\kappa_{u}=-\kappa_{v}=\lambda$. The semi-discretization described above may be easily adapted for arbitrary initial-boundary conditions. For brevity, we present here only the case of homogeneous boundary conditions. For this case we establish numerically the stability of the trivial solution $Y=0$. Parameter values for which instability occurs are found via an eigenproblem for the Jacobian matrix $J$ of the system of ordinary differential equations (33).

It should be noted that preservation of cosymmetry in a finite-difference approximation of a two-dimensional filtration-convection problem on a rectangular domain is considered in $[6,9]$.

\subsection{Computation of the family of equilibria}

If $\lambda$ is slightly greater than $\lambda_{\text {crit }}$, then all points on the family are stable. So, we can start to integrate (33) in an arbitrary non-zero state, and compute the solution. It approaches a limit state which, in general, depends on the initial state. This limit state is not an isolated asymptotically stable one, but one point on a continuous curve of stationary states.

To compute the family of equilibria we apply a technique similar to the approach developed in $[5,7]$. This technique is based on the cosymmetric version of the Implicit Function Theorem, cf. [4], which asserts the existence of a continuous family of equilibria, and states that the kernel of the Jacobian is tangential to the family (curve) in phase space.

We trace this curve consisting of equilibria, defined by

$$
(A+\lambda B) Y+F(Y)=0 .
$$

The Jacobian matrix $J=A+\lambda B+\partial_{Y} F(Y)$ has a constant rank deficiency of one. We find the family of equilibria (stationary solutions) by following the $1 \mathrm{~d}$ solution manifold of this algebraic system of equations by a predictor-corrector method. This means, we cancel one equation of the system (35), adding instead one equation of the form

$$
\left(Y-Y_{e}\right) \cdot Y_{t}=\tau,
$$

where $Y_{e}$ is our actual equilibrium solution and $Y_{t}$ is a unit basis vector of $\operatorname{ker} J, \tau$ is the step-size. The thus modified equilibrium condition defines for small enough $\tau$ a unique new equilibrium point. A reasonable starting point for Newton's method is $Y_{e}+\tau Y_{t}$.

Without going into the details we remark that for higher ratios of $\lambda$ stability of some stationary solutions may be lost. However, we can still trace the family by the above method, finding an initial guess by increasing $\lambda$ gradually.

It should be mentioned that if we take either parameters not satisfying the conditions (9) or we use an approximation which destroys the cosymmetry during discretization, we end up with a set of isolated equilibrium states.

\section{Numerical Results}

Our aim is to verify numerically the existence of a continuous family of equilibria for the system (1)-(3), and at the same time to demonstrate the robustness of the described 
numerical approach.

We take the particular set of parameters

$$
\epsilon_{u}=\epsilon_{v}=0.1, \quad \kappa_{u}=-\kappa_{v}=\lambda, \quad \alpha=2, \quad \beta=4, \quad \gamma=0, \quad \delta=0,
$$

and vary the parameter $\lambda$ to observe the transformation of the family. The computations were done on a grid with $N=40$ nodes. The results on the grid $N=20$ are practically the same. To display the computed families we will use a projection to the plane with coordinates

$$
U=\sum_{j=1}^{N} u_{j}, \quad V=\sum_{j=1}^{N} v_{j} .
$$

In Fig. 1 we present the families for a number of parameter values $\lambda$. It is clearly visible

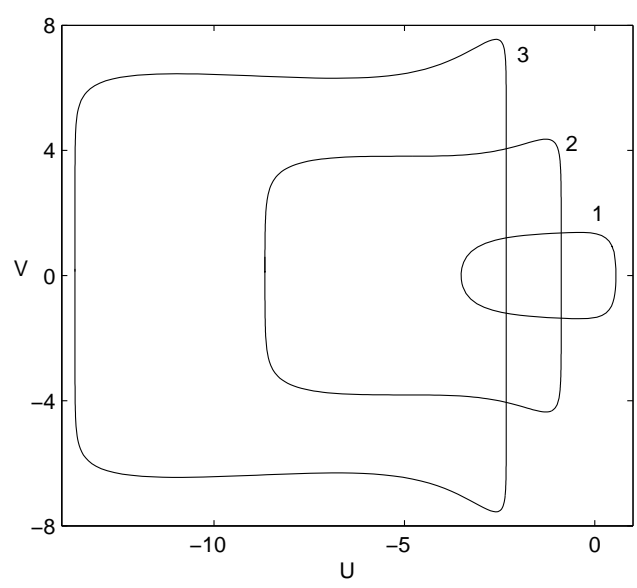

Fig. 1: Families of equilibria for different values $\lambda$.

that the family of equilibria expands and shifts with increasing parameter $\lambda$, and its form changes. We observe symmetry of all curves with respect to the horizontal line $V=0$. This is a consequence of a discrete symmetry which exists for the given choice of parameters. In fact, equation (1) is invariant under the reflection

$$
\{x, u, v\} \mapsto\left\{\frac{a}{2}-x, u,-v\right\} .
$$

In Fig. 2 we present the real parts of a number of greatest eigenvalues from the spectra of equilibria. We parameterized the curve by arc coordinate along the family on $([0,1])$. One can see that the spectrum varies when the point moves along the family. Hence, the computed families are not the result of the action of a symmetry group. We also note the complication of the spectrum with increasing $\lambda$. All states from the families presented in Fig. 1 are transversally stable with respect to the family. There always exists a zero eigenvalue corresponding to the direction tangential to the family. Thus, the direction along the family of equilibria is a neutral one. The first instability on the family occurs at $\lambda=7$.

In Fig. 3 and Fig. 4 we present the distributions of $u(x)$ and $v(x)$ for a number of stationary points on the family. One can see how the steady state varies. The solution for $u(x, t)$ may be symmetric with respect to the center of the interval $[0, a]$ (even function 

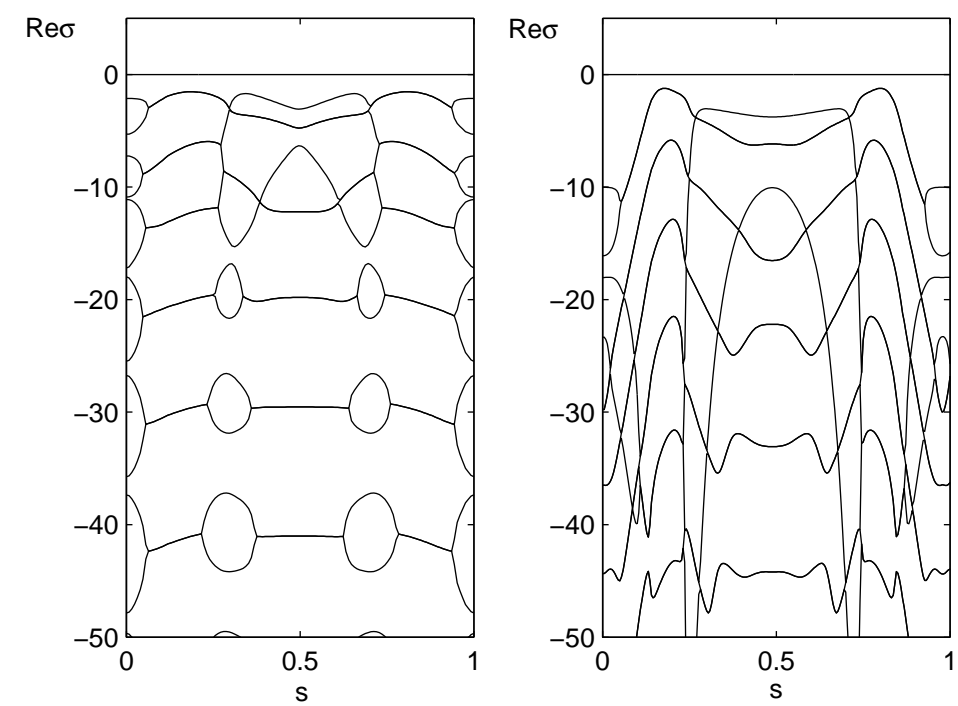

Fig. 2: Real part of spectrum for equilibria on the family; $\lambda=1$ (left) and $\lambda=2$ (right).

w.r.t. $a / 2)$, and the corresponding distribution of $v(x, t)$ is given by a skew-symmetric curve (odd function w.r.t. $a / 2$ ).
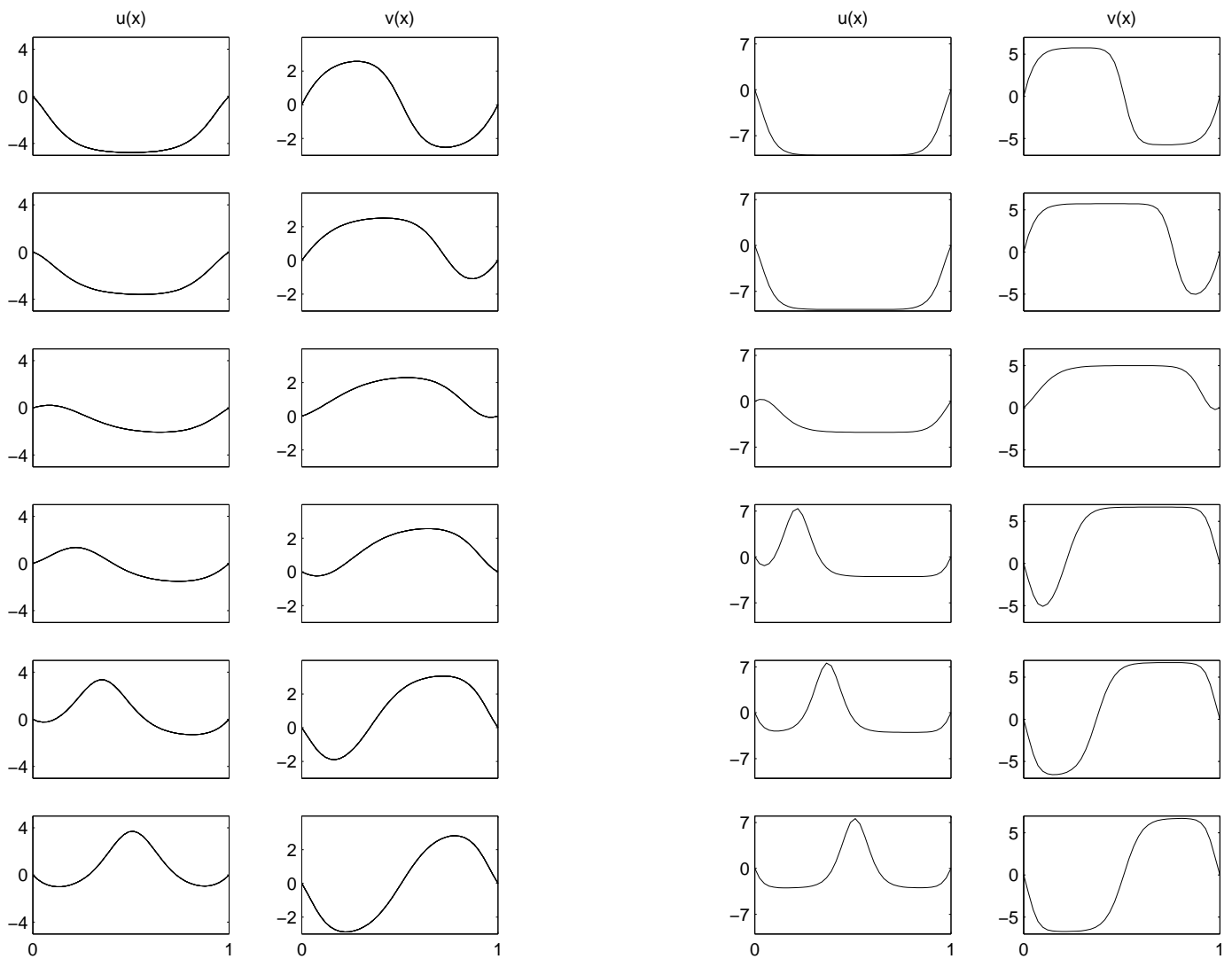

Fig. 3: Selected solutions from the family; $\lambda=1$.
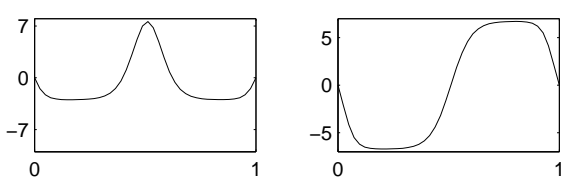

Fig. 4: Selected solutions from the family; $\lambda=2$.

Fig. 5 demonstrates that the equilibria are really accessible through the solution of initial 
value problems. We assume the initial conditions

$$
u_{j}=A \sin \left(\frac{\pi x_{j}}{a}\right), \quad v_{j}=B \sin \left(\frac{\pi x_{j}}{a}\right), \quad j=1 \cdots N,
$$

and solve the system of ordinary differential equations (35) by a Runge-Kutta method on the time span $[0,4]$.

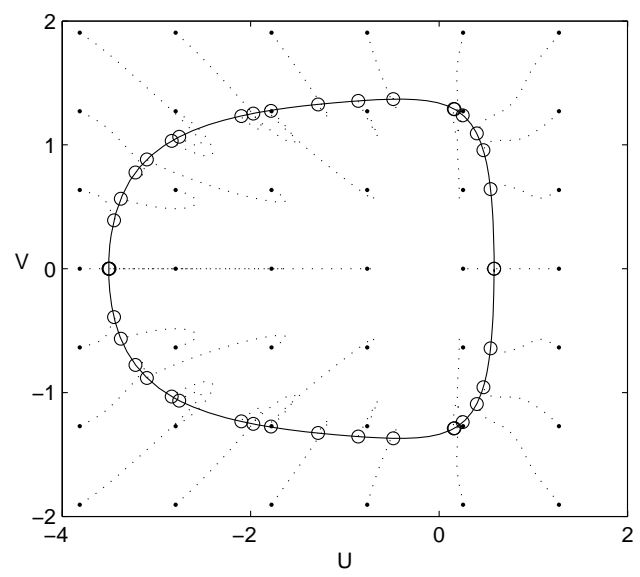

Fig. 5: Attraction to the family of equilibria.

In Figures 5-7 an initial point is marked by a big dot, a trajectory is displayed by a dotted line and a final position is marked by a circle. Because of the symmetry of the initial distribution, the resulting phase portrait keeps the symmetry w.r.t. the line $V=0$.

Note that deviation of parameters away from the requirements of Lemma 1 immediately leads to the destruction of the family - trajectories are attracted to a limited number of isolated equilibrium points. This is illustrated in Fig. 6 and Fig. 7 where we use a value of $M_{22}^{v}$ not equal to -6 as Lemma 1 would imply for the given set of parameters.

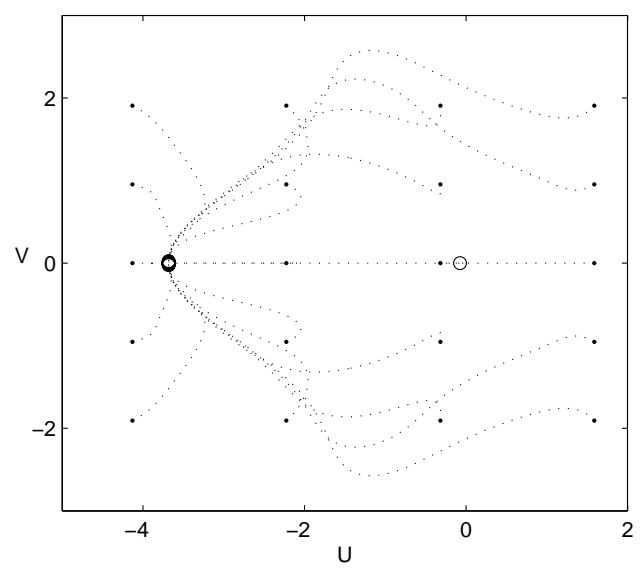

Fig. 6: Destruction of continuity due to loss of cosymmetry; $M_{22}^{v}=-2$.

All trajectories in Fig. $6\left(M_{22}^{v}=-2\right)$ are attracted to two stable isolated equilibria. It is interesting that the transient dynamics reproduces partially the continuous family curve. There is no more a continuous family - but it looks as if the system 'remembered' it. We take time span $T=6$ to get reasonably close to a steady situation in Fig. 6 . 


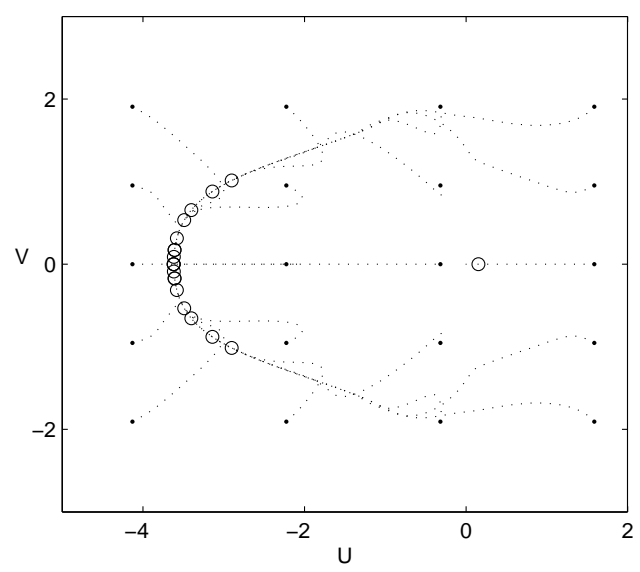

Fig. 7: Destruction of continuouity due to loss of cosymmetry; $M_{22}^{v}=-4$.

In the case $M_{22}^{v}=-4$ the evolution is very slow, see Fig. 7, and one can observe a number of the points are situated practically on the (lost) 'family' curve. Here we see that the time span $T=9$ was insufficient to complete the transient process.

Figures 6 and 7 demonstrate that study of cosymmetry may be very useful for nearly cosymmetrical cases for which the system has only a finite number of isolated equilibria. It provides a better understanding of the dynamics and explains its essential features.

Another reason for the loss of continuity of the family of equilibria may be an inappropriate finite-difference approximation. To demonstrate that we put $\zeta=6$ instead of correct value $\zeta=1$ which preserves the cosymmetry. We take a number points from the family as initial conditions and integrate the system on the time interval $[0,120]$ up to relaxation.
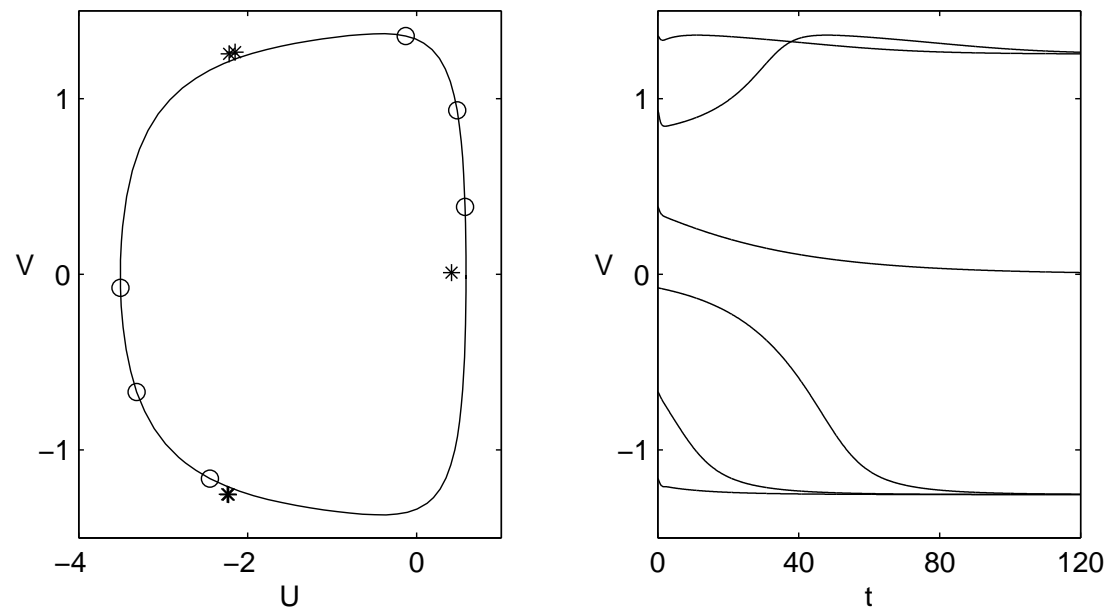

Fig. 8: Destruction of continuity by bad choice of FDM parameter $\zeta=6$.

In the left part of Fig. 8 the initial and final states are marked by circles and stars, respectively. The right part of Fig. 8 gives the dependence of the variable $V$ on $t$. Note that the transient process takes a long time, and moreover, that the trajectories are located close to the family.

Our computations display three stable equilibria in the case of non-adequate approximation of nonlinear terms. It is simple to check that the linear function (7) is not a cosymmetry now, although it vanishes on isolated equilibria. We also detect that there exist three unstable equilibria. 
The equilibrium with $V=0$ has a symmetric distribution on $u(x, t)$ and an antisymmetric one on $v(x, t)$ as shown in the last row in Fig. 3. Two other equilibria form a pair without symmetry, but one of them transforms to the other under reflection w.r.t. $a / 2$ (mirror symmetric pair).

This way, the continuous family of equilibria is transformed to a finite number of isolated equilibria. The number and location of the isolated states depend on the deviation from the parameter $\zeta=1$. We take the same initial points and $\zeta=-4$ and repeat the direct integration. One can see that the location of the equilibria is transformed in comparison with Fig. 8. In this case the destruction of the continuous family also leads to the appearance of three stable and three unstable equilibria lying in the vicinity of the curve corresponding to the lost family.
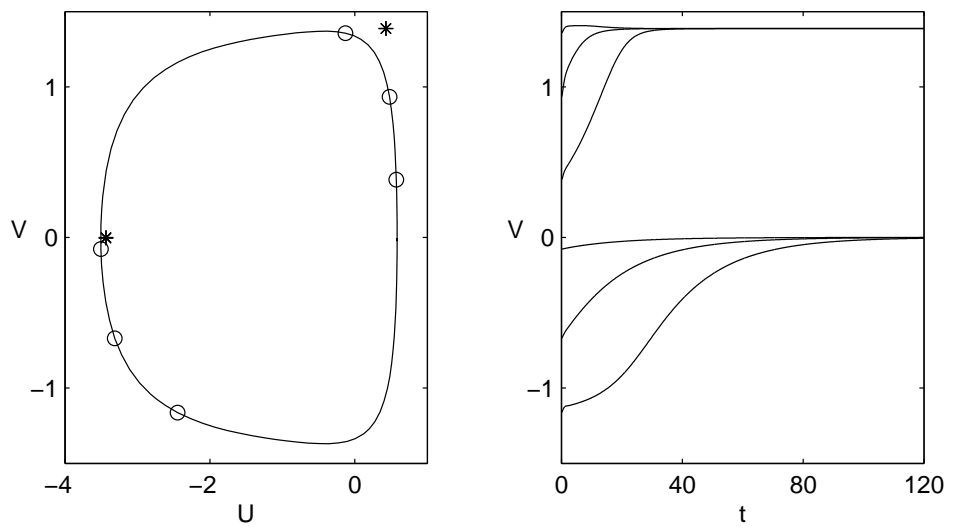

Fig. 9: Destruction of family due to bad FDM parameter $\zeta=-4$

It should be mentioned that for $\zeta$ close to 1 we need a long-time integration to reach a stable equilibrium.

For the application in population kinetics, we note that a trivial equilibrium would assume constant population densities along the whole space. The results presented in this section show that for the special choice of parameters, stable distributions may occur while members of both groups move all the time due to diffusion - in order to level out the distributions. These diffusive fluxes, however, are balanced by the growth/decay caused by the flux of the respective other group, cf. Fig. 3 and Fig. 4.

\section{Conclusion}

Known examples of cosymmetric systems [1-10] include the planar problem of filtration of an incompressible fluid in a saturated porous medium, the abstract Darcy model, and some applications to analytical mechanics.

Here we considered a population model with two species of quite different behavior. A system of two parabolic differential equations was formulated and continuous non-symmetric families of equilibrium states were computed. We intend to extend the proposed model to the case of two and three space dimensions.

Loss of cosymmetry either from inappropriate parameter choice or bad discretization may lead to dynamics without a continuous family of equilibria. However, in a certain sense, the behavior of the systems stays close to one with cosymmetry. Thus, the investigation of the family of equilibria brings insight to the dynamics of the system. 


\section{Acknowledgements}

V. Ts. thanks Rostock University for hospitality during visits in 2001 - 2002, the Russian Foundation for Basic Research (grant no. 01-01-22002) and the program "Russian Universities - Fundamental Research" (project no. UR.04.01.063) for partial support.

\section{References}

[1] Yudovich V. I. Cosymmetry, degeneracy of the solutions of operator equations, and the onset of filtrational convection, Matem. Zametki, 49, 142-160, 1991.

[2] Yudovich V. I. Secondary cycle of equilibria in a system with cosymmetry, its creation by bifurcation and impossibility of symmetric treatment of it, Chaos, 5, 402-441, 1995.

[3] Bratsun D. A. Lyubimov D. V. and Roux B. Co-symmetry breakdown in problems of thermal convection in porous medium, Physica D, 82, 398-417, 1995.

[4] Yudovich V. I. Implicit function theorem for cosymmetric equations, Matem. Zametki, 60, 313-317, 1996.

[5] Govorukhin V. G. Numerical simulation of the loss of stability for secondary steady regimes in the Darcy plane-convection problem, Doklady Akademii Nauk, 363, 806808, 1998.

[6] Karasözen B. Tsybulin V. G. Finite-Difference Approximations and Cosymmetry Conservation in Filtration Convection Problem. Physics Letters, A 262, 321-329, 1999.

[7] Govorukhin V. G. Calculation of one-parameter families of stationary regimes in a cosymmetric case and analysis of plane filtrational convection problem. In Continuation methods in fluid dynamics (Aussois, 1998), Notes Numer. Fluid Mech., 74, Vieweg, Braunschweig, 133-144, 2000.

[8] Kurakin L. G. Yudovich V. I. Bifurcations accompanying monotonic instability of an equilibrium of a cosymmetric dynamical system, Chaos, 10, 311-330, 2000.

[9] Karasözen B. Tsybulin V. G. Conservative Finite Difference Schemes for Cosymmetric Systems. Proc. 4th Conf. on Computer Algebra in Scientific Computing, SpringerVerlag, 363-375, 2001.

[10] Yudovich V. I. Cosymmetry and conservative systems, preprint VINITI. 2001. (in Russian)

[11] Courant R. Hilbert D. Methods of Mathematical Physics, Intersience, New York, Vol. 1, 1953.

[12] Ganzha V. G. and Vorozhtsov E. V. Numerical Solutions for Partial Differential Equations. Problem Solving Using Mathematica. CRC Press, Boca Raton, New York, London, 1996. 